\title{
MiR-93 suppresses cell proliferation and invasion by targeting ZNF322 in human hepatocellular carcinoma
}

\section{Type}

Research paper

\section{Keywords}

hepatocellular carcinoma, cell proliferation, Poor prognosis, miR-93, ZNF322

\begin{abstract}
Introduction

This study aimed to investigate the biological role of microRNA 93 (miR-93), a novel tumor-related miRNA, in human hepatocellular carcinoma ( $\mathrm{HCC}$ ) and elucidate the potential molecular mechanisms involved.

Material and methods

Quantitative real-time polymerase chain reaction (qRT-PCR) was conducted to determine the expression of miR-93 in HCC tissues and cell lines. The log-rank test and Kaplan-Meier survival analysis were performed to evaluate the relationship between miR-93 expression and overall survival. MTT assay, colony formation assay, Transwell migration and invasion assays were carried out to exam cell proliferation, colony formation, migration and invasion, respectively. Murine xenograft models were established to the effect of miR-93 on tumor growth in vivo. TargetScan online software was applied to predict the potential target of miR-93. Luciferase reporter assays were used to validate the direct binding of miR-93 and its putative target.
\end{abstract}

Results

Here we found that miR-93 was significantly down-regulated in HCC tissues and cell lines. Patients with decreased miR-93 expression had a significantly shorter overall survival. Functional investigations demonstrated miR-93 over-expression suppressed HCC cell proliferation, weakened clonogenic ability, and slowed down cell migration and invasion; whereas miR-93 depletion facilitated HCC cell proliferation, colony formation, cell migration and invasion. MiR-93 over-expression retarded tumor growth in vivo. Luciferase reporter assay and rescue assay revealed that zinc finger protein 322 (ZNF322) was a direct target of miR-93 and mediated the inhibitory effects of miR-93 on HCC cell proliferation and motility.

Conclusions

Our data may provide some evidence for miR-93/ZNF322 axis a candidate therapeutic target for HCC. 
MiR-93 suppresses cell proliferation and invasion by targeting ZNF322 in human hepatocellular carcinoma

Running title: MiR-93 functions as a tumor suppressor in HCC 


\section{Abstract}

Introduction: This study aimed to investigate the biological role of microRNA 93 (miR-93), a novel tumor-related miRNA, in human hepatocellular carcinoma (HCC) and elucidate the potential molecular mechanisms involved.

Materials and methods: Quantitative real-time polymerase chain reaction (qRT-PCR) was conducted to determine the expression of miR-93 in HCC tissues and cell lines. The log-rank test and Kaplan-Meier survival analysis were performed to evaluate the relationship between miR-93 expression and overall survival. MTT assay, colony formation assay, Transwell migration and invasion assays were carried out to exam cell proliferation, colony formation, migration and invasion, respectively. Murine xenograft models were established to the effect of miR-93 on tumor growth in vivo. TargetScan online software was applied to predict the potential target of miR-93. Luciferase reporter assays were used to validate the direct binding of miR-93 and its putative target.

Results: Here we found that miR-93 was significantly down-regulated in HCC tissues and cell lines. Patients with decreased miR-93 expression had a significantly shorter overall survival. Functional investigations demonstrated miR-93 over-expression suppressed HCC cell proliferation, weakened clonogenic ability, and slowed down cell migration and invasion; whereas miR-93 depletion facilitated HCC cell proliferation, colony formation, cell migration and invasion. MiR-93 over-expression retarded tumor growth in vivo. Luciferase reporter assay and rescue assay revealed that zinc finger protein 322 (ZNF322) was a direct target of miR-93 and mediated the inhibitory effects of miR-93 on HCC cell proliferation and motility.

Conclusions: Our data may provide some evidence for miR-93/ZNF322 axis a candidate therapeutic target for HCC. 
Keywords: hepatocellular carcinoma, miR-93, cell proliferation, poor prognosis, ZNF322

\section{Introduction}

Hepatocellular carcinoma (HCC) is one of the major causes of tumor-related morbidity and mortality, resulting in more than 600,000 deaths worldwide [1-3]. It was estimated approximately 40,000 new HCC cases were diagnosed and around 28,000 patients died of HCC in United States in 2017 [4]. It is documented that drug resistance and malignant metastasis is partially responsible for the high mortality of HCC patients [5-7]. Despite the fact that significant breakthroughs have been achieved in diagnosis and treatment, the long-term prognosis remains rather depressing in HCC patients. Therefore, there is an urgent demand to develop effective therapeutic strategies for HCC.

MicroRNAs (miRNAs) are a large class of small ( 22 nucleotides) non-coding RNA molecules that negatively regulate gene expression at post-transcription level by binding to the 3' untranslated regions (3'UTRs) of their target messenger RNAs (mRNAs) [8-10]. Mounting evidence has revealed that miRNAs function as critical regulators in the carcinogenesis and tumor progression [11-14]. Recent studies have demonstrated that abnormal expression of miRNAs contributes to the occurrence and development of diverse types of human malignancies [15-18]. MicroRNA 93 (miR-93), a novel tumor-associated miRNA, has been identified to be implicated in multiple types of human neoplasms, including breast cancer [19], colorectal cancer [20, 21], laryngeal squamous cell carcinoma [22] and nasopharyngeal carcinoma [23]. Nonetheless, the biological role of miR-93 in HCC remains largely obscure.

Zinc finger proteins constitute the largest family of transcription factors in eukaryotes [24, 25]. Zinc finger protein 322 (ZNF322) belongs to the large family of 
zinc finger transcription factors [26]. Previous studies have demonstrated that ZNF322 serves as an oncogene in multiple types of human malignant tumors.

In the present study, we found that miR-93 was significantly down-regulated in HCC tissues compared with adjacent normal tissues. Functional investigations showed that miR-93 suppressed HCC cell proliferation, migration and invasion. Furthermore, mechanistic studies revealed that miR-93 exerted its inhibitory effects on HCC proliferation and motility partially through targeting ZNF322.

\section{Material and methods}

\section{Patients and tissue samples}

58 paired tumorous and non-tumorous tissues were collected from HCC patients who underwent surgical resection at the Second Affiliated Hospital of Xi'an Jiaotong University (Xi'an, China) between March 2011 and December 2016. None of patients received chemotherapy, immunotherapy or radiotherapy before. All the tissue samples were stored at $-80^{\circ} \mathrm{C}$ until further studies. Clinicopathological parameters of $\mathrm{HCC}$ patients were listed in Table 1. Overall survival time was defined as the interval between the date of primary surgery treatment and the date of death or last follow-up. This study was approved by the Ethics Committee of Xi'an Jiaotong University (Xi'an, China). All the patients gave their written consents.

\section{Cell lines and cell culture}

Normal human liver L0 cells and four HCC cell lines (HepG2, Huh6, Huh7 and Hep3B) were purchased from Shanghai Cell Bank of Chinese Academy of Sciences (Shanghai, China). All the cells were cultured in Dulbecco's modified Eagle's medium (DMEM; Gibco, Grand Island, NY, USA) supplemented with 10\% fetal bovine serum (FBS; Gibco). The cells were maintained at $37^{\circ} \mathrm{C}$ in a humidified incubator containing $5 \% \mathrm{CO}_{2}$. 


\section{Cell Transfection}

Cell transfection was carried out using Lipofectamine 2000 (Invitrogen Life Technologies, Carlsbad, CA, USA) according to the manufacturer's protocol. Sequences of miR-93 mimics and miR-93 inhibitor were designed and synthesized by GenePharma Co. Ltd (Shanghai, China). The sequence information was listed as followed: miR-93 mimics, 5'-CAAAGTGCGACCCGTGCAGGTAG-3' ; miR-93 inhibitor, 5'-CTACCTGCACGGGTCGCACTTTG-3'. The cells were harvested for further studies at $48 \mathrm{~h}$ post-transfection. The transfection efficiency was evaluated using quantitative real-time polymerase chain reaction.

\section{Cell proliferation assays}

Cell proliferation was assessed using MTT Cell Proliferation Assay Kit (Thermo Fisher Scientific, San Jose, CA, USA) according to the manufacturer's instructions as previously described [27]. In brief, $20 \mu 1$ MTT solution (Merck Millipore, Billerica, MA, USA) at a concentration of $5 \mathrm{mg} / \mathrm{mL}$ was added to each well of 96 -well culture plate and incubated for another 4 hours at $37^{\circ} \mathrm{C}$. The supernatants were discarded and $150 \mu \mathrm{l}$ DMSO was added to the cells. The absorbance was measured at $570 \mathrm{~nm}$ using a microplate reader (Bio-Tek Instruments, Winooski, VT, USA). All the experiments were performed in triplicate.

\section{Colony formation assays}

Clonogenic abilities of HCC cells were determined by colony formation assays. Briefly, cells were seed on $60 \mathrm{~mm}$-dish $\left(1 \times 10^{3}\right.$ cells per dish) and cultured for two weeks. The colonies were stained using $0.1 \%$ crystal violet (Sigma Aldrich, St. Louis, MO, USA) for $5 \mathrm{~min}$ after fixation with $10 \%$ formaldehyde for $15 \mathrm{~min}$. Visible colonies were counted under an invert light microscope (Nikon, Tokyo, Japan).

Transwell migration and invasion assays 
HCC cell migration and invasion capabilities were determined by Transwell migration and invasion assays using 24-well transwells ( $8 \mu \mathrm{m}$ pore size; Millipore) according to the manufacturer's protocol. For migration assays, about $1 \times 10^{4}$ cells were seeded into the upper chambers containing FBS-free DMEM medium. The lower chambers were filled with DMEM containing 10\% FBS. The Transwell plates were then incubated at $37{ }^{\circ} \mathrm{C}$ for 24 hours. Then the cells remaining on the upper chamber were removed with cotton wool. Cells that migrated through the membrane were fixed in $4 \%$ para-formaldehyde and stained with $0.5 \%$ crystal violet (Sigma) for $30 \mathrm{~min}$. Then the migrated cells from five random visual fields were counted under a light microscope (Nikon) and photographed at a magnification of 400X. For invasion assays, the membrane was coated with Matrigel (BD Bioscience, San Jose, CA, USA). And the procedures of Transwell invasion assays were in accordance with migration assays.

\section{Western blotting analysis}

Total proteins were extracted using RIPA buffer supplemented with protease inhibitor cocktail (Roche, Basel, Switzerland). The protein samples were separated using SDS-polyacrylamide gel electrophoresis (PAGE) and then transferred onto the polyvinylidene fluoride PVDF membranes. After being blocked with 5\% degrease milk in TBST buffer, the membranes were incubated with primary antibodies overnight at $4^{\circ} \mathrm{C}$. Anti-ZNF322 (ab155519), anti-MMP9 (ab137867) and anti-vimentin (ab45939) and anti-GAPDH (ab125247) were purchased from Abcam (Cambridge, MA, USA). Antibodies were used at the following dilutions: anti-ZNF322 (1:1000), anti-MMP9 (1:1000), anti-vimentin (1:1000) and anti-GAPDH (1:2000). After being washed three times, the membranes were then incubated with horseradish peroxidase (HRP)-labeled secondary antibody at $37^{\circ} \mathrm{C}$ for $1 \mathrm{~h}$. The blots were developed using the enhanced chemiluminescence (ECL) Western Blotting 
Detection Kit (Amersham, Buckinghamshire, England) and visualized on a Gel Doc XR System (Bio-Rad Laboratories, Hercules, CA, USA).

Quantitative real-time polymerase chain reaction

Total RNA was extracted using Trizol Reagent in accordance with manufacturer's instruction. For miRNA expression analysis, miR-93 was reverse-transcribed using TaqMan MiRNA Reverse Transcription Kit (Applied Biosystems, Foster, CA, USA). U6 was used as an endogenous control to normalize miR-93 expression. For ZNF322 mRNA expression, the first stand was synthesized using TaqMan High-Capacity cDNA Reverse Transcription Kit (Applied Biosystems). GAPDH was used as internal control for normalization of ZNF322 mRNA expression. PCR was performed on an Applied Biosystems 7500 Fast Real-time PCR system. The sequences of specific primers were listed as followed: miR-93, forward 5'-GCCAGATCTGCACTGTGGGTACTTG-3' and reverse 5'-GCCGAATTCGCACTGTGGGTACTGT-3'; $\quad$ ZNF322, forward 5'-AGAGAGCCTGCAATTGGAAA-3' and reverse 5'-ACGAGAAGGGCTGAGCATTA-3'; $\quad$ GAPDH, forward 5'-GACTCATGACCACAGTCCATGC-3' and reverse 5'-AGAGGCAGGGATGATGTTCTG-3'; U6, forward 5'-CTCGCTTCGGCAGCACA-3' and reverse 5'-AACGCTTCACGAATTTGCGT-3'. The relative transcript abundance was determined according to the $2^{-\triangle \Delta \mathrm{Ct}}$ method. All the experiments were carried out in triplicate.

Immunohistochemical (IHC) analysis

Paraffin-embedded tissues were sectioned at $4.5 \mu \mathrm{m}$ thickness. After being dewaxed and hydrated, sections were incubated with $3 \% \mathrm{H} 2 \mathrm{O} 2$ for 30 min to block the endogenous peroxidase (POD) activity. Following antigen recovery by repeated 
cooling and heating, 5\% bovine serum albumin (BSA) was applied to block non-specific binding. The sections were then incubated with primary antibodies overnight at $4^{\circ} \mathrm{C}$. Anti-ZNF322 (ab155519) was purchased from Abcam and used at a dilution of 1:500. After being rinsed with PBS three times for 5 min each, slices were treated with biotinylated secondary antibody (Abcam) for $1 \mathrm{~h}$, followed by incubation with streptavidin-horseradish peroxidase (HRP) for $20 \mathrm{~min}$. Diaminobenzidine (DAB) substrate was used to visualize ZNF322-positive cells. Slides were then observed under a microscope (Nikon).

\section{Luciferase reporter assays}

Luciferase reporter assays were used to validate the direct binding of miR-93 and ZNF322 mRNA 3'UTR fragments. In brief, the wild-type ZNF322 mRNA 3'UTR fragments containing the predicted binding site of miR-93 were amplified from human genomic DNA by PCR using specific primers. The mutant ZNF322 mRNA 3'UTR fragments were constructed by site-directed mutagenesis using a QuikChange Site-Directed Mutagenesis Kit (Stratagene, La Jolla, CA, USA) according to the manufacturer's instructions. The wild-type and mutant ZNF322 mRNA 3'UTR fragments were cloned into the pGL3-basic dual luciferase reporter vectors (Promega, Madison, WI, USA), respectively. The luciferase activity of the reporter vectors carrying wild-type or mutant ZNF322 mRNA 3'UTR fragments was analyzed in the presence of miR-93. A dual-luciferase reporter system (BD Bioscience) was used to determine the relative luciferase activity at 48 hour post-transfection. Firefly luciferase activity was normalized to Renilla luciferase activity.

\section{Tumorigenicity in nude mice}

Male BALB/c nude mice (5-6 weeks of age, $\mathrm{n}=12$ ) were purchased from Guangdong

Medical Laboratory Center (Guangzhou, China) for the establishment of the 
subcutaneous xenograft tumor models. Briefly, NC mimics or miR-93 mimics-treated Hep3B cells $\left(5 \times 10^{6}\right.$ cells per mouse $)$ were subcutaneously injected into the flanks of the nude mice. Tumor size was measured using slide caliper every five days, and tumor volumes were calculated according to the formula:volume $\left(\mathrm{mm}^{3}\right)=\left[\right.$ width $^{2}$ $\left(\mathrm{mm}^{2}\right) \times$ length $\left.(\mathrm{mm})\right] / 2$. At day 35 post-inoculation, all the nude mice were sacrificed, and the tumors were collected and weighted. This animal protocol was approved by Animal Care and Use Committee of Xi'an Jiaotong University (Xi'an, China).

\section{Statistical analysis}

Data were presented as mean \pm standard deviation. Statistical analysis was conducted using SPSS 18.0 software (SPSS Inc., Chicago, IL, USA). Student's $t$-test was used to compare the difference between two groups. One-way analysis of variance (ANOVA) followed by the Dunnett's multiple comparisons was applied to analyze the differences among three independent groups. Fisher's exact test was employed to evaluate the relationship between miR-93 expression and clinicopathological characteristics of patients. The log-rank test and Kaplan-Meier survival analysis were used to assess miR-93 expression and overall survival. Pearson's correlation analysis was applied to determine the correlation between miR-93 expression and ZNF322 mRNA expression in tumorous tissues. $P<0.05$ was considered statistically significant.

\section{Results}

\section{Decreased miR-93 expression predicts poor prognosis of HCC patients}

Even though miR-93 has been identified as a critical regulator in the tumorigenesis and tumor progression of multiple types of human malignant neoplasms, its biological role in HCC remains largely unclear. To investigate the biological role of miR-93 in HCC, we determined its expression levels in 58 pairs of tumorous tissues and matched 
non-tumorous tissues. As presented in Figure 1A, HCC tissues displayed lower miR-93 expression levels than adjacent non-cancerous tissues. To evaluate the correlation between miR-93 expression and clinicopathological characteristics of HCC patients, tumorous tissues were divided into high miR-93 expression group and low miR-93 expression. Fisher's exact test demonstrated that decreased miR-93 expression was associated with larger tumor size, advanced TNM stage and lymph node metastasis (Table 1). The log-rank test and Kaplan-Meier survival analysis showed that HCC patients with low miR-93 expression experienced a significantly shorter overall survival than those with high miR-93 expression (Figure 1B). Consistently, we found that miR-93 was significantly down-regulated in four HCC cell lines (HepG2, Huh6, Huh7 and Hep3B) compared with normal human liver cell line L02 (Figure 1C). Collectively, these findings indicate that decreased miR-93 expression correlates with poor prognosis of HCC patients.

\section{MiR-93 suppresses HCC cell proliferation and colony formation}

Given the findings mentioned above, we speculated that miR-93 was involved in hepatocellular carcinogenesis and tumor progression. To better understand the biological role of miR-93 in HCC, we transfected HCC cells with miR-93 mimics or miR-93 inhibitor to manipulate its expression. Over-expression and knockdown studies were conducted in Hep3B cells (lowest endogenous miR-93 expression) and Hep3B cells (highest endogenous miR-93 expression), respectively. Transfection efficiency was evaluated by qRT-PCR analysis (Figure 2A). As evident from MTT assays, miR-93 over-expression significantly inhibited Hep3B cell proliferation compared with negative control group, whereas miR-93 depletion notably facilitated HepG2 cell proliferation (Figure 2B). To explore the effect of miR-93 on HCC cell growth in vivo, we established mouse xenograft models by subcutaneous injection of 
NC mimics or miR-93 mimics-treated Hep3B cells. As presented in Figure 2C, tumors collected from miR-93 over-expression group weighed significantly lighter than those from negative control group; moreover, tumors formed by miR-93 mimics-treated Hep3B cells grew dramatically slower than those formed by NC mimics-treated Hep3B cells. As exhibited in Figure 2D, miR-93 over-expression significantly suppressed the clonogenic capabilities of Hep3B cells compared with negative control treatment, whereas miR-93 ablation dramatically enhanced the clonogenic abilities of Hep2G cells. Taken together, these data suggest that miR-93 suppresses HCC proliferation and colony formation.

\section{MiR-93 inhibits HCC cell migration and invasion}

Cell migration and invasion are crucial steps in tumor malignant progression. To evaluate whether miR-93 affects the motility of HCC cells, we carried out Transwell migration and invasion assays. As displayed in Figure $\mathbf{3 A}$ and $\mathbf{3 B}$, miR-93 over-expression significantly weakened the migration and invasion abilities of Hep3B cells in comparison with negative control group, while miR-93 knockdown sharply strengthened the migration and invasion capabilities of HepG2 cells. Furthermore, western blotting analysis showed that expression of vimentin and MMP9 proteins was significantly increased in miR-93 mimics-treated Hep3B cells compared with negative control group, whereas miR-93 depletion remarkably inhibited the expression of vimentin and MMP9 proteins (Figure 3C). Our findings indicate that miR-93 represses HCC cell migration and invasion.

\section{ZNF322 is a direct target of miR-93 in HCC cells}

To clarify the potential molecular mechanisms by which miR-93 inhibited HCC cell proliferation, migration and invasion, we carried out bio-informatics analysis using TargetScan online software. Among all the putative targets, ZNF322 caught our 
attention for its crucial role in carcinogenesis and was selected as a candidate target of miR-93 (Figure 4A). To verify whether miR-93 could bind to to 3'UTR of ZNF322 mRNA, we performed luciferase reporter assays. As shown in Figure 4B, transfection of miR-93 mimics significantly decreased the activity of luciferase reporter vectors carrying wild-type 3'UTR fragments, whereas transfection of miR-93 mimics failed to trigger significant changes in the activity of luciferase reporter vectors carrying mutant 3'UTR fragments. Moreover, qRT-PCR and western blotting analyses demonstrated that miR-93 mimics significantly elevated ZNF322 mRNA and protein expression levels compared with negative control treatment, while miR-93 ablation remarkably reduced the expression levels of ZNF322 mRNA and protein (Figure 4C and 4D). Notably, we found that tumorous tissues exhibited higher ZNF322 expression levels than matched non-cancerous tissues (Figure 4E). Besides, Pearson's correlation analysis showed that miR-93 expression levels were negatively correlated with ZNF322 mRNA expression levels in HCC tissues (Figure 4F). To sum up, our results suggest that ZNF322 is a downstream direct target of miR-93 in HCC cells.

Rescue of ZNF322 expression reverses the inhibitory effects of miR-93 mimics on HCC cell proliferation, migration and invasion

To analyze the function connection between miR-93 and ZNF322, we rescued the expression of ZNF322 in miR-93 mimics-treated Hep3B cells (Figure 5A). As presented in Figure 5B-D, restoration of ZNF322 expression alleviated miR-93 mimics-induced suppressive effects on Hep3B cell proliferation, migration and invasion. Furthermore, western blotting analysis demonstrated that rescue of ZNF322 expression reversed the inhibitory effects of miR-93 mimics on the expression of vimentin and MMP9 proteins (Figure 5E). Collectively, our data suggest that miR-93 exerts its inhibitory effects on HCC proliferation and motility by directly targeting 
ZNF322.

\section{Discussion}

HCC is the fifth most common human malignant tumor and ranks the third leading cause of cancer-related deaths worldwide [28-30]. It is widely acknowledged that HCC has posed great threats to human life and imposed great pressures on public health around the globe $[31,32]$. Growing evidence has revealed that miRNAs serve as critical regulators in the tumorigenesis and their aberrant expression leads to the malignant progression of human neoplasms [15-18]. Numerous studies have reported that abnormal expression of miRNAs contributes to hepatocellular carcinogenesis [33-35]. In spite of great advances in the diagnosis and treatment of HCC over the past several decades, the long-term prognosis of patients remains very poor. Hence, it is imperative to seek novel diagnostic bio-markers and develop effective therapeutic strategies for HCC.

Previous studies have shown that miR-93 was dys-regulated and involved in multiple types of human cancers. Liu et al reported that miR-93 was significantly down-regulated and exerted tumor-suppressing functions in breast cancer cells [19]. Yang et al demonstrated that miR-93 inhibited tumor growth and early relapse of human colorectal cancer [20]. Chen et al revealed that miR-93-5p inhibited tumorigenesis and progression in human epithelial ovarian cancer by targeting RhoC [36]. Xiao et al found that miR-93 was significantly down-regulated in human colon cancer tissues and that decreased miR-93 expression was associated with poor prognosis of patients [37]. However, the biological role of miR-93 in HCC is still poorly understood. In the current study, we found that miR-93 was significantly down-regulated in HCC tissues and that decreased miR-93 expression correlated with poor prognosis of patients. Taken together, these findings indicate the potential of 
miR-93 as a tumor suppressor in hepatocellular carcinogenesis.

To better understand the biological role of miR-93 in HCC, we subsequently carried out functional studies. Gain- and loss-of function analyses were used to conduct functional investigations. Our results demonstrated that miR-93 over-expression suppressed HCC cell proliferation, migration and invasion, whereas miR-93 depletion accelerated cell proliferation, migration and invasion. Furthermore, we found that miR-93 over-expression repressed tumor growth in mouse xenograft models. Collectively, our data suggest that miR-93 possesses anti-tumor activity in vitro and in vivo.

To elucidate the potential molecular mechanisms by which miR-93 exerts its inhibitory effects on HCC proliferation, migration and invasion, we performed bio-informatics analysis using TargetScan online algorithms and chose ZNF322 as a candidate target of miR-93. Moreover, luciferase reporter assays identified ZNF322 as a downstream direct target of miR-93. It is well documented that ZNF322 was a member of the large zinc finger family of transcription factors [38]. Previous studies have reported that ZNF322 exerted oncogenic functions by regulating tumor-associated gene expression and activating tumorigenic signaling pathway [39, 40]. Elevated expression of ZNF322 has been demonstrated to contribute to occurrence and development of gastric cancer [41] and lung cancer [42].

To validate the function link between between miR-93 and ZNF322, we rescued the expression of ZNF322 in miR-93 mimics-treated Hep3B cells. Interestingly, we found that restoration of ZNF322 expression partially reverse the suppressive effects of miR-93 over-expression on Hep3B cell proliferation and motility. To sum up, these results suggest that miR-93 exerts its tumor-suppressing functions in $\mathrm{HCC}$ by directly targeting ZNF322. 
To conclude, the present study for the first time demonstrated that miR-93 was significantly down-regulated in $\mathrm{HCC}$ and that decreased miR-93 expression was associated with poor prognosis of patients. Moreover, mechanistic studies unveiled that miR-93 exerted its anti-cancer activity in HCC by targeting ZNF322. The current study provides new insights into understanding the potential molecular mechanisms underlying hepatocellular carcinogenesis and tumor progression. Thus, our study indicate that targeting miR-93/ZNF322 axis may be used as a promising therapy for HCC.

\section{Acknowledgements}

This study was supported by Natural Science Basic Research Plan in Shaanxi Province of China (2014JM2-3034).

\section{Disclosure of conflict of interests}

None.

\section{References}

1. Elserag HB, Rudolph KL. Hepatocellular carcinoma: epidemiology and molecular carcinogenesis. Gastroenterology 132:2557-2576, 2007.

2. Murakami Y, Yasuda T, Saigo K, Urashima T, Toyoda H, Okanoue T, Shimotohno K. Comprehensive analysis of microRNA expression patterns in hepatocellular carcinoma and non-tumorous tissues. Oncogene, 25: 2537-2545, 2006.

3. Gish RG, Lencioni R, Di Bisceglie AM, Raoul JL, Mazzaferro V. Role of the multidisciplinary team in the diagnosis and treatment of hepatocellular carcinoma. Expert Rev Gastroenterol Hepatol 6: 173-185, 2012.

4. Siegel RL, Miller K D , Jemal A . Cancer Statistics, 2017. Ca Cancer J Clin 67:7-30, 2017. 
5. Cheng BY, Lau EY, Leung HW, Leung CO, Ho NP, Gurung S, Cheng LK, Lin CH, Lo RC, Ma S, Ng IO, Lee TK. IRAK1 Augments Cancer Stemness and Drug Resistance via the AP-1/AKR1B10 Signaling Cascade in Hepatocellular Carcinoma. Cancer Res 78: 2332-2342, 2018.

6. Li T, Xie J, Shen C, Cheng D, Shi Y, Wu Z, Deng X, Chen H, Shen B, Peng C, Li H, Zhan Q, Zhu Z. Upregulation of long noncoding RNA ZEB1-AS1 promotes tumor metastasis and predicts poor prognosis in hepatocellular carcinoma. Oncogene 35: 1575-1584, 2016.

7. Zhou SL, Hu ZQ, Zhou ZJ, Dai Z, Wang Z, Cao Y, Fan J, Huang XW, Zhou J. miR-28-5p-IL-34-macrophage feedback loop modulates hepatocellular carcinoma metastasis. Hepatology 63: 1560-1575, 2016.

8. Godshalk SE, Bhaduri-Mcintosh S, Slack FJ. Epstein-Barr virus-mediated dysregulation of human microRNA expression. Cell Cycle 7: 3595-3600, 2008.

9. Xie Q, Chen X, Lu F, Zhang T, Hao M, Wang Y, Zhao J, Mccrae MA, Zhuang H. Aberrant expression of microRNA 155 may accelerate cell proliferation by targeting sex-determining region Y box 6 in hepatocellular carcinoma. Cancer 118: 2431-2442, 2012.

10. Wang J, He J, Su F, Ding N, Hu W, Yao B, Wang W, Zhou G. Repression of ATR pathway by miR-185 enhances radiation-induced apoptosis and proliferation inhibition. Cell Death Dis 4: e699, 2013.

11. Suzuki H I , Katsura A, Matsuyama H, et al. MicroRNA regulons in tumor microenvironment. Oncogene 34: 3085-3094, 2015. 
12. Cheng Z, Wang HZ, Li X, Wu Z, Han Y, Li Y, Chen G, Xie X, Huang Y, Du Z, Zhou Y. MicroRNA-184 inhibits cell proliferation and invasion, and specifically targets TNFAIP2 in Glioma. J Exp Clin Cancer Res 34: 27, 2015.

13. Su Y, Li X, Ji W, Sun B, Xu C, Li Z, Qian G, Su C. Small molecule with big role: MicroRNAs in cancer metastatic micro-environments. Cancer Lett 344: 147-156, 2014.

14. Cao Q, Liu F, Ji K, Liu N, He Y, Zhang W, Wang L. MicroRNA-381 inhibits the metastasis of gastric cancer by targeting TMEM16A expression. J Exp Clin Cancer Res 36: 29, 2017.

15. Mizuguchi Y, Takizawa T, Yoshida H, Uchida E. Dysregulated miRNA in progression of hepatocellular carcinoma: A systematic review. Hepatol Res 46: 391-406, 2016.

16. Yang X, Zhang XF, Lu X, Jia HL, Liang L, Dong QZ, Ye QH, Qin LX. MicroRNA-26a suppresses angiogenesis in human hepatocellular carcinoma by targeting hepatocyte growth factor-cMet pathway. Hepatology 59: 1874-1885, 2014.

17. Chang RM, Yang H, Fang F, Xu JF, Yang LY. MicroRNA-331-3p promotes proliferation and metastasis of hepatocellular carcinoma by targeting PH domain and leucine-rich repeat protein phosphatase. Hepatology 60: 1251-1263, 2014.

18. Bae HJ, Noh JH, Kim JK, Eun JW, Jung KH, Kim MG, Chang YG, Shen Q, Kim SJ, Park WS, Lee JY, Nam SW. MicroRNA-29c functions as a tumor suppressor by direct targeting oncogenic SIRT1 in hepatocellular carcinoma. Oncogene 33: 2557-2567, 2014. 
19. Liu S, Patel SH, Ginestier C, Ibarra I, Martin-Trevino R, Bai S, Mcdermott SP, Shang L, Ke J, Ou SJ, Heath A, Zhang KJ, Korkaya H, Clouthier SG, Charafe-Jauffret E, Birnbaum D, Hannon GJ, Wicha MS. MicroRNA93 regulates proliferation and differentiation of normal and malignant breast stem cells. PLoS Genet 8: e1002751, 2012.

20. Yang IP, Tsai HL, Hou MF, Chen KC, Tsai PC, Huang SW, Chou WW, Wang JY, Juo SH. MicroRNA-93 inhibits tumor growth and early relapse of human colorectal cancer by affecting genes involved in the cell cycle. Carcinogenesis 33: 1522-1530, 2012.

21. Tang Q, Zou Z, Zou C, Zhang Q, Huang R, Guan X, Li Q, Han Z, Wang D, Wei H, Gao X, Wang X. MicroRNA-93 suppress colorectal cancer development via Wnt/beta-catenin pathway downregulating. Tumour Biol 36: 1701-1710, 2015.

22. Xiao X, Zhou L, Cao P, Gong H, Zhang Y. MicroRNA-93 regulates cyclin G2 expression and plays an oncogenic role in laryngeal squamous cell carcinoma. Int $\mathbf{J}$ Oncol 46: 161-174, 2015.

23. Xu YF, Mao YP, Li YQ, Ren XY, He QM, Tang XR, Sun Y, Liu N, Ma J. MicroRNA-93 promotes cell growth and invasion in nasopharyngeal carcinoma by targeting disabled homolog-2. Cancer Lett 363: 146-155, 2015.

24. Meruvu S, Hugendubler L, Mueller E. Regulation of adipocyte differentiation by the zinc finger protein ZNF638. J Biol Chem 286: 26516-26523, 2011.

25. Liu J, Stormo GD. Context-dependent DNA recognition code for C2H2 zinc-finger transcription factors. Bioinformatics 24: 1850-1857, 2008. 
26. Ma H, Ng HM, Teh X, Li H, Lee Y H, Chong YM, Loh YH, Collins JJ, Feng B, Yang H, Wu Q. Zfp322a Regulates mouse ES cell pluripotency and enhances reprogramming efficiency. PLoS Genet 10: e1004038, 2014.

27. Li JY, Liu CP, Shiao WC, Jayakumar T, Li YS, Chang NC, Huang SY, Hsieh CY. Inhibitory effect of PDGF-BB and serum-stimulated responses in vascular smooth muscle cell proliferation by hinokitiol via up-regulation of p21 and p53. Arch Med Sci 14: 579-587, 2018.

28. Dhanasekaran R, Limaye A, Cabrera R. Hepatocellular carcinoma: current trends in worldwide epidemiology, risk factors, diagnosis, and therapeutics. Hepat Med 4: 19-37, 2012.

29. Kasprzak A, Rogacki K, Adamek A, Sterzynska K, Przybyszewska W, Seraszek-Jaros A, Helak-Lapaj C, Pyda P. Tissue expression of beta-catenin and Eand N-cadherins in chronic hepatitis $\mathrm{C}$ and hepatocellular carcinoma. Arch Med Sci 13: 1269-1280, 2017.

30. Schutte K, Bornschein J, Malfertheiner P. Hepatocellular carcinoma--epidemiological trends and risk factors. Dig Dis 27: 80-92, 2009.

31. Chen G, Li X, Yang J, Li J, Wang X, He J, Huang Z. Prognostic significance of cyclooxygenase-2 expression in patients with hepatocellular carcinoma: a meta-analysis. Arch Med Sci 12: 1110-1117, 2016.

32. Yue Y, Yang Y, Shi L, Wang Z. Suppression of human hepatocellular cancer cell proliferation by Brucea javanica oil-loaded liposomes via induction of apoptosis. Arch Med Sci 11: 856-862, 2015. 
33. Borel F, Konstantinova P, Jansen PL. Diagnostic and therapeutic potential of miRNA signatures in patients with hepatocellular carcinoma. J Hepatol 56: 1371-1383, 2012.

34. Zhang J, Jin H, Liu H, Lv S, Wang B, Wang R, Liu H, Ding M, Yang Y, Li L, Zhang J, Fu S, Xie D, Wu M, Zhou W, Qian Q. MiRNA-99a directly regulates AGO2 through translational repression in hepatocellular carcinoma. Oncogenesis 3: e97, 2014.

35. Chang RM, Xiao S, Lei X, Yang H, Fang F, Yang LY. miRNA-487a Promotes Proliferation and Metastasis in Hepatocellular Carcinoma. Clin Cancer Res 23: 2593-2604, 2017.

36. Chen X, Chen S, Xiu YL, Sun KX, Zong ZH, Zhao Y. RhoC is a major target of microRNA-93-5P in epithelial ovarian carcinoma tumorigenesis and progression. Mol Cancer, 14: 31, 2015.

37. Xiao ZG, Deng ZS, Zhang YD, Zhang Y, Huang ZC. Clinical significance of microRNA-93 downregulation in human colon cancer. Eur J Gastroenterol Hepatol 25: 296-301, 2013.

38. Xia L, Delomenie C, David I, Rainer Q, Marouard M, Delacroix H, David DJ, Gardier AM, Guilloux JP. Ventral hippocampal molecular pathways and impaired neurogenesis associated with 5-HT(1)A and 5-HT(1)B receptors disruption in mice. Neurosci Lett 521: 20-25, 2012.

39. Jen J, Lin LL, Chen HT, Liao SY, Lo FY, Tang YA, Su WC, Salgia R, Hsu CL, Huang HC, Juan HF, Wang YC. Oncoprotein ZNF322A transcriptionally deregulates 
alpha-adducin, cyclin D1 and p53 to promote tumor growth and metastasis in lung cancer. Oncogene 35: 2357-2369, 2016.

40. Li Y, Wang Y, Zhang C, Yuan W, Wang J, Zhu C, Chen L, Huang W, Zeng W, Wu X, Liu M. ZNF322, a novel human C2H2 Kruppel-like zinc-finger protein, regulates transcriptional activation in MAPK signaling pathways. Biochem Biophys Res Commun 325: 1383-1392, 2004.

41. Hu X, Zhang M, Miao J, Wang X, Huang C. miRNA-4317 suppresses human gastric cancer cell proliferation by targeting ZNF322. Cell Biol Int 42: 923-930, 2018. 42. Liao SY, Chiang CW, Hsu CH, Chen YT, Jen J, Juan HF, Lai WW, Wang YC. CK1delta/GSK3beta/FBXW7alpha axis promotes degradation of the ZNF322A oncoprotein to suppress lung cancer progression. Oncogene 36: 5722-5733, 2017. 


$$
\text { Low }(\mathrm{n}=30) \quad \text { High }(\mathrm{n}=28)
$$

Age

$<60$

$\geqq 60$

Gender

Female

Male

HBV infection

Yes

No

Tumor size $(\mathrm{cm})$

$<5$

$\geqq 5$

Liver cirrhosis

Yes

No

Lymph node metastasis

Yes

25

No

TNM stage

I, II

III, IV

29

29

31

27

27

31

26

32
17

23

10

0.002

7

18

Table 1. Correlation between miR-93 expression and clinicopathological parameters of HCC patients 
Figure 1. Decreased miR-93 expression predicts poor prognosis of $\mathrm{HCC}$ patients. (A) Relative expression levels of miR-93 in 58 paired tumorous tissues and adjacent non-cancerous tissues were determined by qRT-PCR analysis. (B) HCC tissues were assigned into high miR-93 expression group and low miR-93 expression group based on the the average value of its expression levels. The log-rank test and Kaplan-Meier survival analysis was used to evaluate the relationship between miR-93 expression and clinicopathological characteristics of HCC patients. (C) Relative expression levels of miR-93 in four HCC cell lines (HepG2, Huh6, Huh7 and Hep3B) and normal human liver L0 cells were detected using qRT-PCR analysis. $* * P<0.01$. HCC, hepatocellular carcinoma; qRT-PCR, quantitative real-time polymerase chain reaction.

Figure 2. MiR-93 suppresses HCC proliferation and colony formation. (A) Transfection efficiency was assessed by qRT-PCR analysis. (B) Cell proliferation was examined by MTT assays after transfection with miR-93 mimics or miR-93 inhibitor. (C) MiR-93 mimics-treated Hep3B cells were subcutaneously injected into the flanks of the nude mice $(n=6)$. Tumor size was measured using slide caliper every 5 days. Mice were sacrificed at day 35 post-implantation, and tumors were weighted. (D) Clonogenic capabilities of HCC cells were determined by colony formation assays after transfection with miR-93 mimics or miR-93 inhibitor. $* * P<0.01$. HCC, hepatocellular carcinoma; qRT-PCR, quantitative real-time polymerase chain reaction; NC, negative control.

Figure 3. MiR-93 inhibits HCC cell migration and invasion. (A) Cell migration was evaluated by Transwell migration assays after transfection with miR-93 mimics or miR-93 inhibitor. (B) Cell invasion was assessed by Transwell invasion assays after transwell invasion assays. (C) Expression levels of vimentin and MMP9 proteins in HCC cells were detected by western blotting assays after transfection with miR-93 
mimics or miR-93 inhibitor. $* * P<0.01$. HCC, hepatocellular carcinoma; $\mathrm{NC}$, negative control; MMP9, matrix metalloprotein 9.

Figure 4. ZNF322 is a direct target of miR-322 in HCC cells. (A) A putative binding site of miR-93 in 3'UTR of ZNF322 mRNA was predicted by TargetScan online software. (B) Luciferase activity of the reporter vectors carrying wild-type or mutant ZNF322 mRNA 3'UTR fragments was examined after transfection with NC mimics or miR-93 mimics. (C) ZNF322 mRNA expression was detected by qRT-PCR analysis after transfection with miR-93 mimics or miR-93 inhibitor. (D) ZNF322 protein expression was assessed by western blotting after transfection with miR-93 mimics or miR-93 inhibitor. (E) ZNF322 protein expression in cancerous tissues and matched para-cancerous tissues was visualized by IHC staining. (F) Pearson's correlation analysis was applied to evaluate the relationship between miR-93 expression and ZNF322 mRNA expression in HCC tissues. $* * P<0.01$. HCC, hepatocellular carcinoma; 3'UTR, 3' untranslated region; ZNF322, zinc finger protein 322; IHC, immunohistochemistry; NC, negative control.

Figure 5. Rescue of ZNF322 expression reverses the inhibitory effects of miR-93 mimics on HCC proliferation, migration and invasion. (A) ZNF322 protein expression was examined by western blotting after rescue of ZNF322 in miR-93 mimics-treated Hep3B cells. (B) Cell proliferation was determined by MTT assays after rescue of ZNF322 in miR-93 mimics-treated Hep3B cells. (C) Cell migration was evaluated by Transwell migration assays after rescue of ZNF322 in miR-93 mimics-treated Hep3B cells. (D) Cell invasion was analyzed by Transwell invasion assays after rescue of ZNF322 in miR-93 mimics-treated Hep3B cells. (E) Expression levels of vimentin and MMP9 were determined by western blotting after rescue of ZNF322 in miR-93 mimics-treated Hep3B cells. $* * P<0.01$. HCC, hepatocellular carcinoma; ZNF322, 
zinc finger protein 322; NC, negative control; MMP9, matrix metalloprotein 9. 

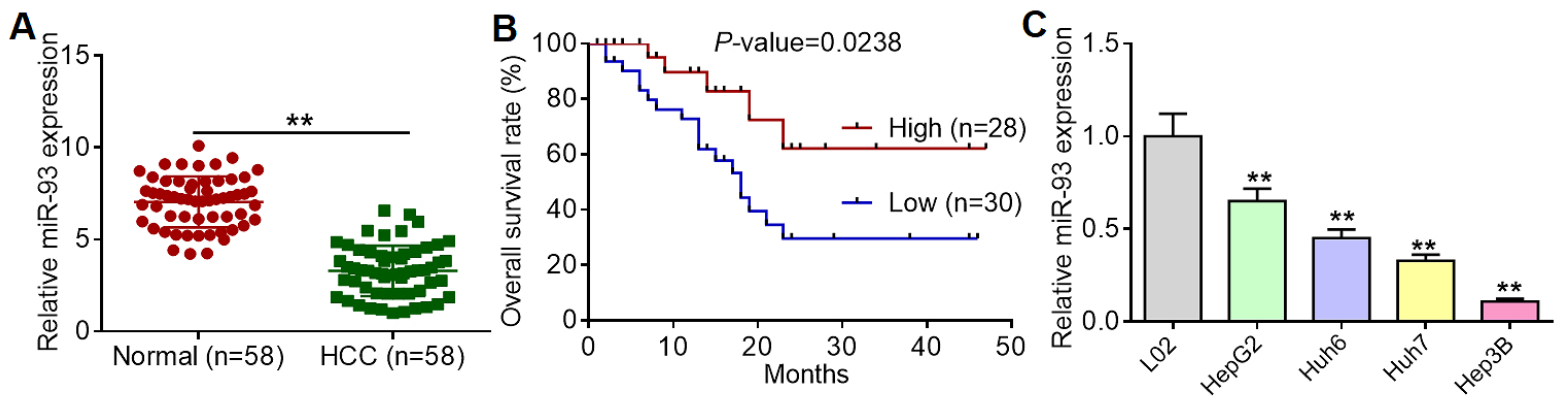

Figure 1 
A

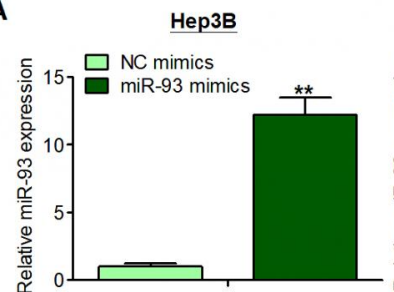

C

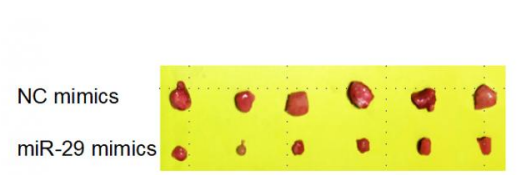

D

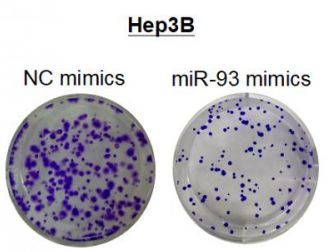

HepG2

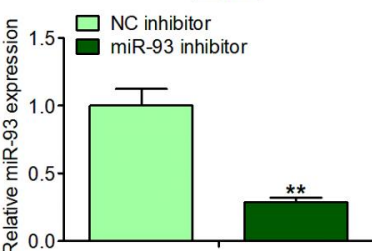

B
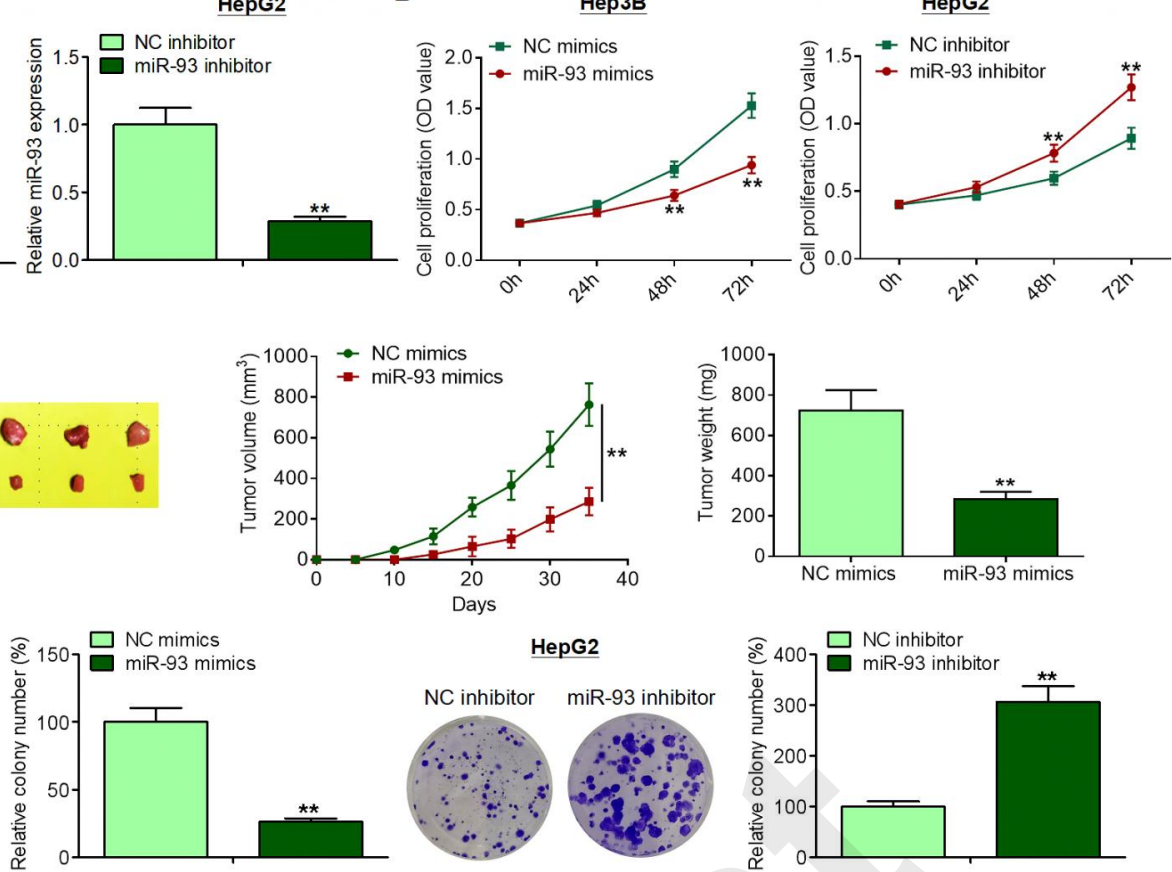
A

Hep3B

NC mimics miR-93 mimics

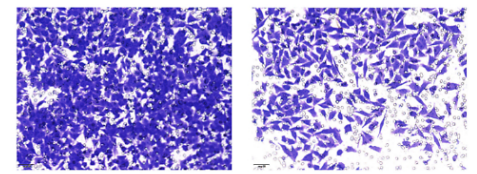

@ $150, \square$ NC mimics

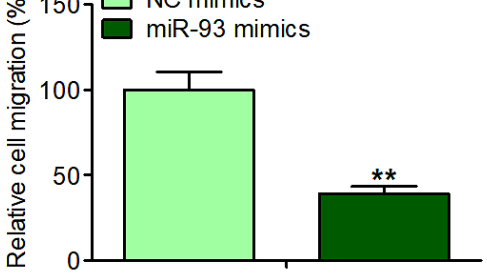

B
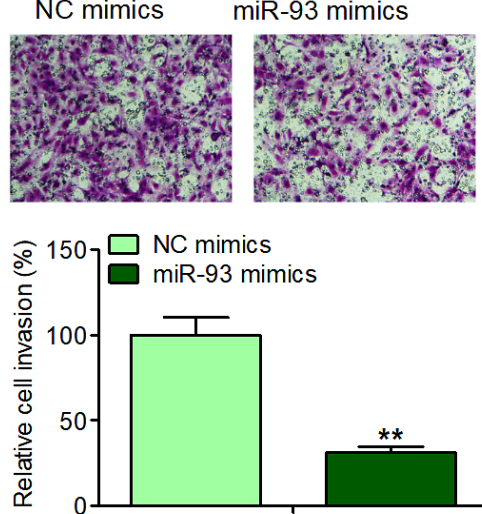

C
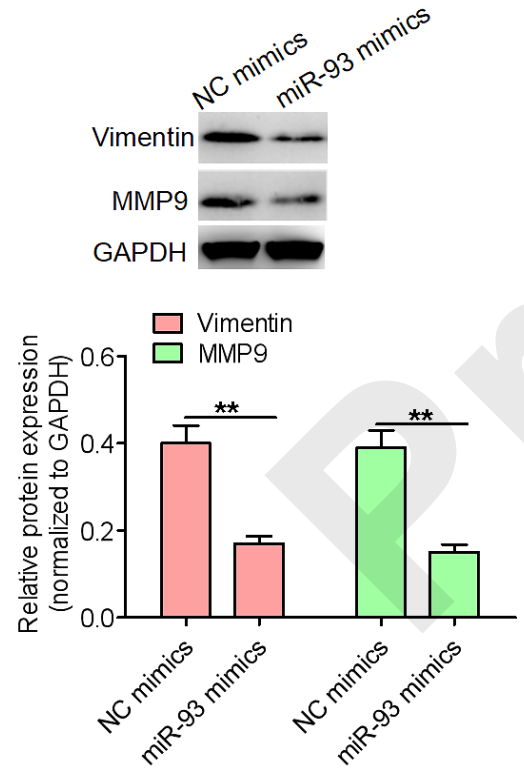
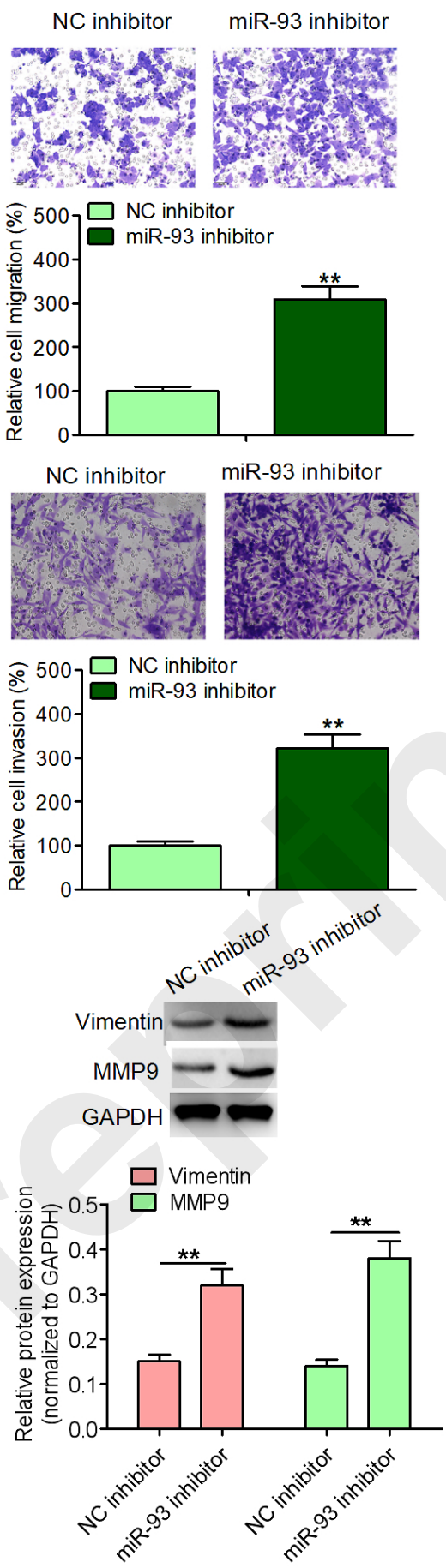

Vimentin -

MMP9 -

GAPDH

Figure 3 
A

wild-type ZNF322 3'UTR 5'-CUUAUCAUCUCUUUCAGCAG-3' I I I I I I I

hsa-miR-93 3'-CCUUCACGAUCGAGUCGUC-5' mutant ZNF322 3'UTR 5'-CUUAUCAUCUCUUAGUCGUC-3'

C

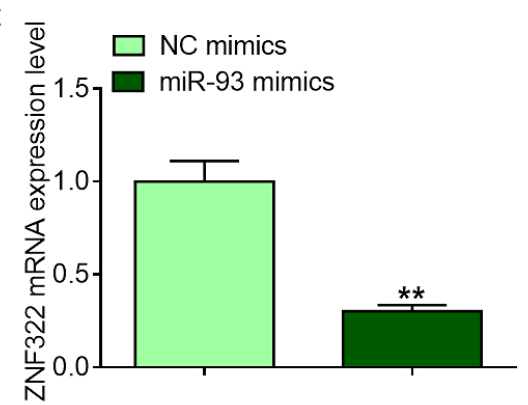

D
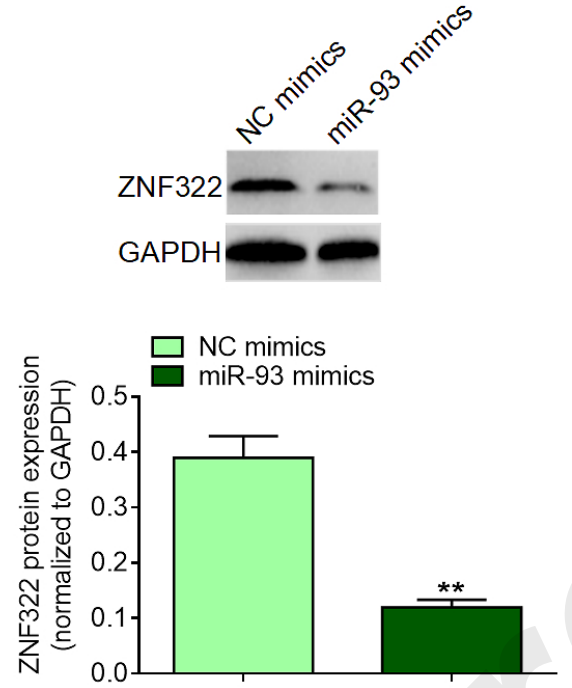

E

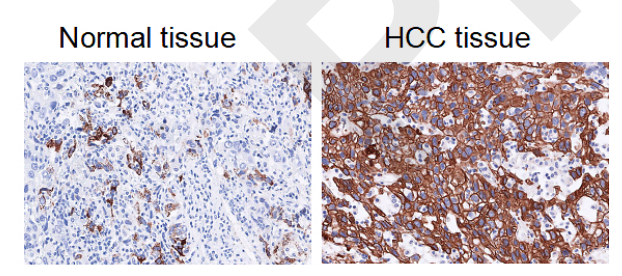

B
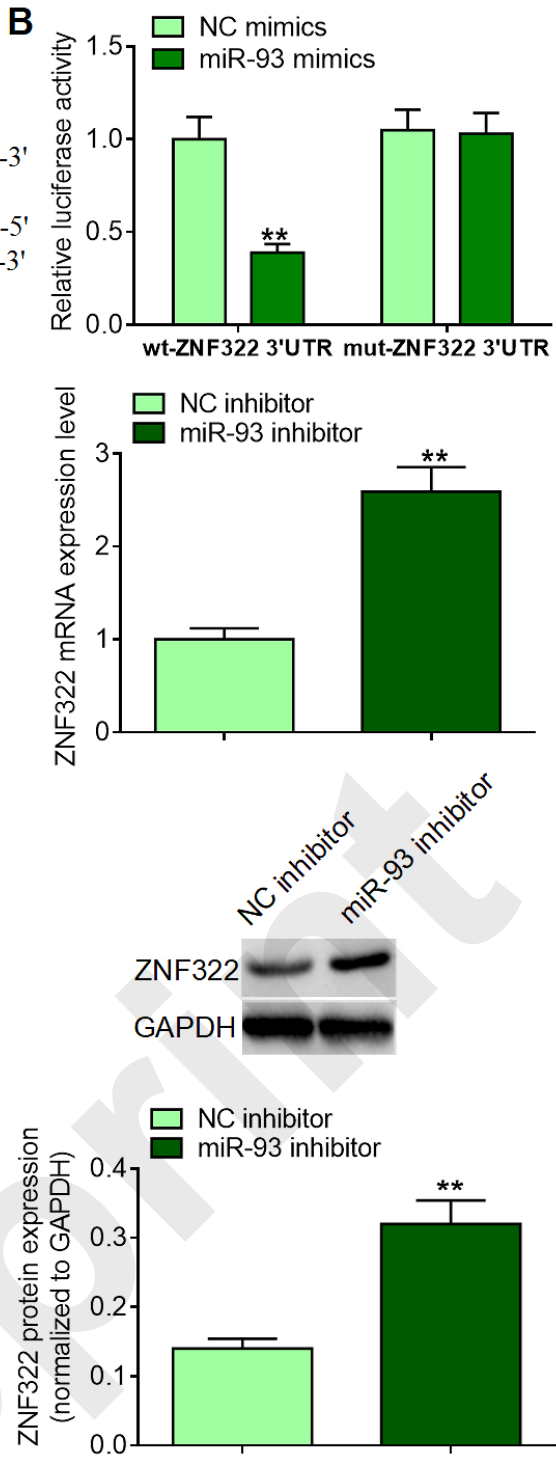

F

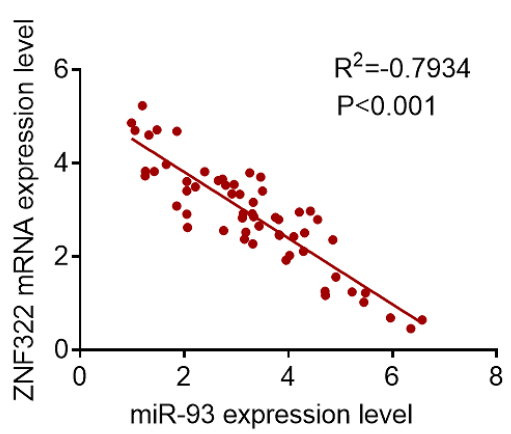

Figure 4 
A

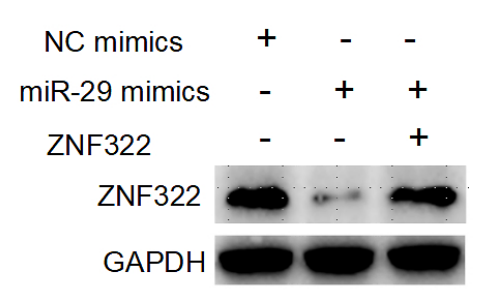

C

NC mimics +

miR-93 mimics

ZNF322

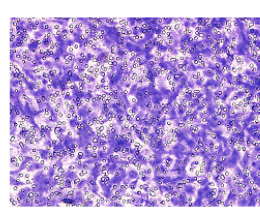

B
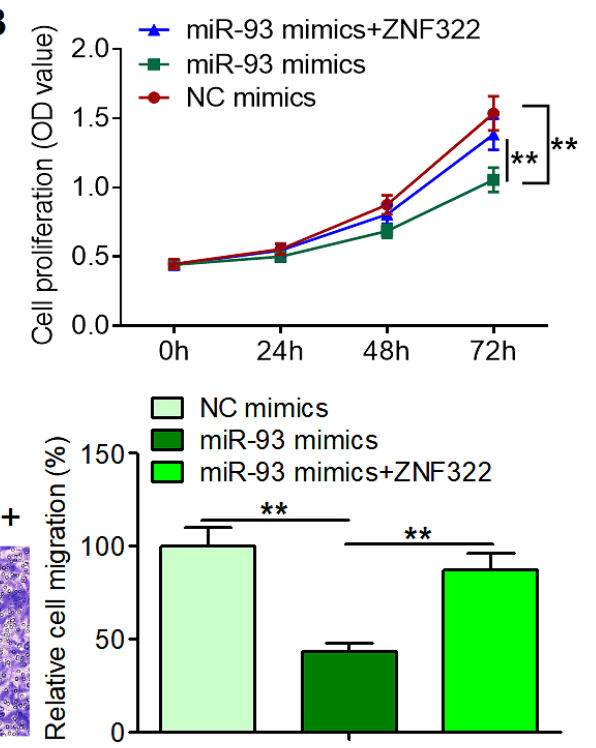

D
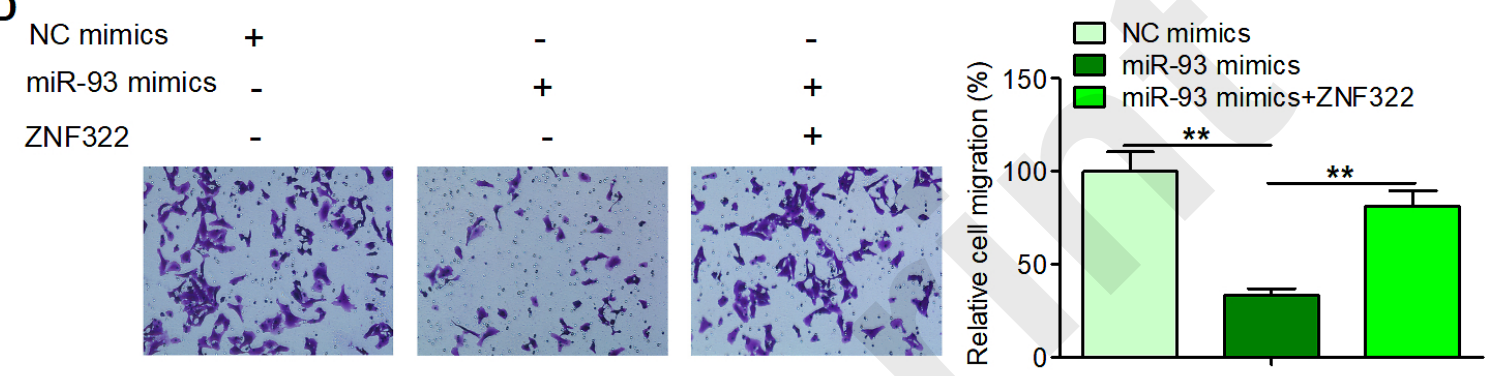

E

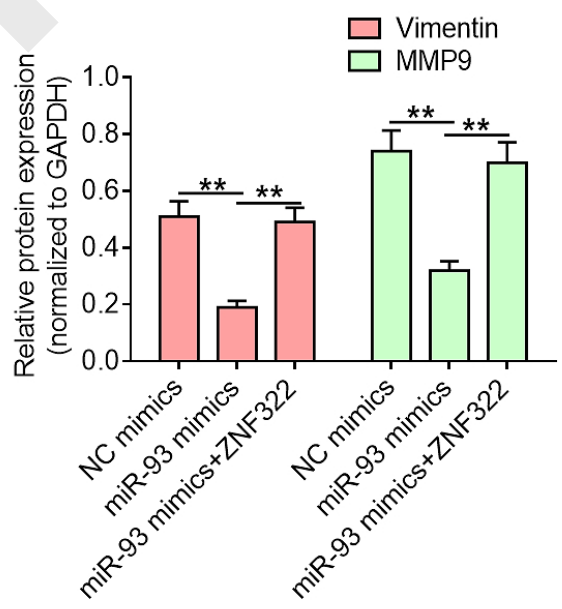

Figure 5 